\title{
High-Redshift Star-Forming Galaxies: Angular Momentum and Baryon Fraction, Turbulent Pressure Effects and the Origin of Turbulence
}

\author{
A. Burkert ${ }^{1,2}$, R. Genzel ${ }^{3}$, N. Bouché ${ }^{3}$, G. Cresci $^{3}$, S. Khochfar ${ }^{3}$, J. Sommer-Larsen ${ }^{4,5}$, A. \\ Sternberg ${ }^{6}$, T. Naab ${ }^{1}$, N. Förster Schreiber ${ }^{3}$, L. Tacconi ${ }^{3}$, K. Shapiro ${ }^{7}$, E. Hicks ${ }^{3}$, D. Lutz ${ }^{3}$, R. \\ Davies $^{3}$, P. Buschkamp ${ }^{3}$, S. Genel ${ }^{3}$ \\ burkert@usm.uni-muenchen.de, genzel@mpe.mpg.de
}

\begin{abstract}
The structure of a sample of high-redshift $(z \sim 2)$, rotating galaxies with high star formation rates and turbulent gas velocities of $\sigma \approx 40-80 \mathrm{~km} / \mathrm{s}$ is investigated. Fitting the observed disk rotational velocities and radii with a Mo, Mao \& White (1998) (MMW) model requires unusually large disk spin parameters $\lambda_{d}>0.1$ and disk-to-dark halo mass fractions of $\mathrm{m}_{d} \approx 0.2$, close to the cosmic baryon fraction. The galaxies segregate into dispersion-dominated systems with $1 \leq v_{\max } / \sigma \leq 3$, maximum rotational velocities $v_{\max } \leq 200 \mathrm{~km} / \mathrm{s}$ and disk half-light radii $r_{1 / 2} \approx 1-3 \mathrm{kpc}$ and rotationdominated systems with $v_{\max }>200 \mathrm{~km} / \mathrm{s}, v_{\max } / \sigma>3$ and $r_{1 / 2} \approx 4-8 \mathrm{kpc}$. For the dispersion-dominated sample radial pressure gradients partly compensate the gravitational force, reducing the rotational velocities. Including this pressure effect in the MMW model, dispersion-dominated galaxies can be fitted well with spin parameters of $\lambda_{d}=0.03-0.05$ for high disk mass fractions of $\mathrm{m}_{d} \approx 0.2$ and with $\lambda_{d}=0.01-0.03$ for $\mathrm{m}_{d} \approx 0.05$. These values are in good agreement with cosmological expectations. For the rotation-dominated sample however pressure effects are small and better agreement with theoretically expected disk spin parameters can only be achieved if the dark halo mass contribution in the visible disk regime $\left(2-3 \times r_{1 / 2}\right)$ is smaller than predicted by the MMW model. We argue that these galaxies can still be embedded in standard cold dark matter halos if the halos did not contract adiabatically in response to disk formation. In this case, the data favors models with small disk mass fractions of $\mathrm{m}_{d}=0.05$ and
\end{abstract}

\footnotetext{
${ }^{1}$ University Observatory Munich (USM), Scheinerstrasse 1, 81679 Munich, Germany

${ }^{2}$ Max-Planck-Fellow

${ }^{3}$ Max-Planck-Institut für extraterrestrische Physik (MPE), Giessenbachstr. 1, 85748 Garching, Germany

${ }^{4}$ Dark Cosmology Centre, Niels Bohr Institute, University of Copenhagen, Juliane Marie Vej 30, 2100 Copenhagen, Denmark

${ }^{5}$ Excellence Cluster Universe, Technical University Munich, Boltzmannstr. 2, D-85748 Garching, Germany

${ }^{6}$ School of Physics and Astronomy, Tel Aviv University, Tel Aviv 69978, Israel

${ }^{7}$ Department of Astronomy, Campbell Hall, University of California, Berkeley, CA 94720, USA
} 
disk spin parameters of $\lambda_{d} \approx 0.035$. It is shown that the observed high turbulent gas motions of the galaxies are consistent with a Toomre instability parameter $\mathrm{Q}=1$ which is equal to the critical value, expected for gravitational disk instability to be the major driver of turbulence. The dominant energy source of turbulence is then the potential energy of the gas in the disk.

Subject headings: cosmology: observations - galaxies: high-redshift - galaxies: individual (BzK-15504) - galaxies: formation - galaxies: evolution - galaxies: halos

\section{Introduction}

Deep surveys have become efficient in detecting star-forming galaxy populations at $\mathrm{z} \sim 1.5-3.5$, near the peak of cosmic star formation, the assembly of massive galaxies and QSO activity (e.g. (Steidel et al. 1996, 2004; Franx et al. 2003; Daddi et al. 2004b)). Large samples are now available, based on their rest-frame UV, or optical, magnitude/color properties. These high-redshift galaxies have star formation rates of $10-300 \mathrm{M}_{\odot} / \mathrm{yr}$, with a range of ages (10 Myrs - 3 Gyrs), stellar masses of $\mathrm{M}_{*} \sim 10^{9}-10^{11.5} \mathrm{M}_{\odot}$ (Shapley et al. 2005; Förster Schreiber et al. 2006; Erb et al. 2006a, b; Daddi et al. 2004a, b) and high gas fractions (Tacconi et al. 2010). They contribute a large fraction of the cosmic star formation activity and stellar mass density at $\mathrm{z} \sim 2$ (Reddv et al. 2005; Rudnick et al. 2006; van Dokkum et al. 2006; Grazian et al. 2007; Pérez-Gonzalez et al. 2008). The majority of these galaxies appears to form stars with high rates over a significant fraction of the $\mathrm{z} \sim 1.5$-3 redshift range (Daddi et al. 2007; Noeske et al. 2007). This requires an efficient and semi-continuous replenishment of fresh gas, perhaps delivered by cold flows/streams from the halo (Dekel \& Birnboim 2006; Dekel et al. 2009a; Dekel. Sari \& Ceverino 2009b; Kereš et al. 2005; Ocvirk, Pichon \& Teyssier 2008; Genzel et al. 2008; Elmegreen \& Burkert 2010; Aumer et al. 2010).

High resolution near-infrared integral field spectroscopy of $\mathrm{H} \alpha$ line emission has shown that most of these high-z star forming galaxies are clumpy and exhibit large ionized gas velocity dispersions of 30-120 km/s (Förster Schreiber et al. 2006, 2009; Genzel et al. 2006, 2008; Law et al. 2007, 2009; Wright et al. 2007, 2009; Van Starkenburg et al. 2008; Stark et al. 2008; Bournaud et al. 2008; Epinat et al. 2009; Cresci et al. 2009). About one third appear to be rotating disks, one third are dispersion dominated systems and one third show clear evidence for interactions and major mergers (Shapiro et al. 2008; Förster Schreiber et al. 2009). The fraction of large, clumpy rotating disks increases with mass. The ratio of the rotational to random velocities ranges between 1 and 6 , quite in contrast to $\mathrm{z} \sim 0$ disk galaxies where $\mathrm{v} / \sigma \sim 10-20$ (Dib. Bell \& Burkert 2006). Many high-z disks are turbulent and geometrically thick (e.g. Elmegreen \& Elmegreen (2006)). Important questions are what drives and maintains these large turbulent velocities and how turbulence is connected to the clumpy disk substructure and the high star formation rates (Immeli et al. $2004 \mathrm{a}, \mathrm{b}$; \begin{tabular}{|l|l|l|}
\hline Bournaud, Elmegreen \& Elmegreen 2007; Bournaud et al. 2008; Dekel et al. 2009a; Dekel, Sari \& Ceverino \\
\hline
\end{tabular} 
2009b; Khochfar \& Silk 2009).

In addition to the unusual kinematics and structure of high-redshift disks, their global physical parameters appear to be puzzling and inconsistent with theoretical expectations. Bournaud, Elmegreen \& Elmegre (2007) found that many high-z galaxies lie in a similar part of the rotation velocity versus disk radius plane as late-type $\mathrm{z} \sim 0$ disks (Courteau et al. 2007) which is not expected according to the standard Mo, Mao \& White (1998) (MMW) model of galactic disk structure. Förster Schreiber et al. (2009) compared the derived dynamical masses with the stellar masses (from spectral energy distribution analysis) and gas masses (from an application of the Kennicutt-Schmidt star formation relation). They found disk masses that are remarkably and perhaps implausibly high.

The MMW model neglects galactic disk turbulence which is reasonable for present day disks with $\mathrm{v} / \sigma \sim 20$. The situation is however different at high redshifts where turbulence can strongly affect the disk structure. This paper discusses the impact of large turbulent motions on the interpretation of the dynamical data of disk galaxies. We show that, including turbulent pressure, the disk spin parameters and disk mass fractions of dispersion-dominated galaxies are reduced to values that are consistent with theoretical expectations. The situation is different for rotation-dominated galaxies where pressure effects play a minor role. As already suggested by numerous studies at low redshifts (e.g. Mo \& Mao (2000); Dutton et al. (2007)), we argue that the observed high-redshift galaxies are in better accord with cosmological models if it is assumed that their dark-matter halos did not contract adiabatically. Finally we propose an explanation for why large turbulence might be more common in many high-z disks and what the energy source of turbulence in these disks might be.

\section{Rotation Curves of Pressurized, Turbulent Galactic Disks}

Let us consider a turbulent galactic gas disk. We analyse its rotational velocity $v_{\text {rot }}$ in the midplane, applying the hydrostatic equation

$$
\frac{v_{r o t}^{2}}{r}=f_{g}(r)+\frac{1}{\rho} \frac{d p}{d r}
$$

where $r$ is the distance from the galactic center and $f_{g}$ is the value of the gravitational force. $p$ is the pressure which consists of a turbulent (kinetic) and thermal part, $p=\rho\left(\sigma^{2}+c_{s}^{2}\right)$ with $\rho$ the gas density, $\sigma$ the characteristic 1-dimensional velocity dispersion of the gas which we assume to be isotropic and $c_{s}$ its sound speed. We define the zero-pressure velocity curve $v_{0}(r)$ as the rotational velocity of the gas if pressure gradients are negligible, i.e. $\mathrm{dp} / \mathrm{dr}=0: v_{0}^{2} \equiv f_{g} \times r$. Equation 1 then reduces to

$$
v_{r o t}^{2}=v_{0}^{2}+\frac{r}{\rho} \frac{d p}{d r}=v_{0}^{2}+\frac{1}{\rho} \frac{d}{d l n r}\left(\rho \sigma^{2}\right)
$$


Here we have neglected the thermal pressure term as the sound speed is in general much smaller than the turbulent velocity. Equation 2 is the most general form, without specifying the radial dependence of $\sigma$ and $\rho$. It demonstrates that a negative radial pressure gradient reduces the rotational velocity of the gas as part of the gravitational force is balanced by the pressure force.

To illustrate the possible importance of pressure effects, let us now assume that $\sigma$ is independent of $r$. Then

$$
v_{\text {rot }}^{2}=v_{0}^{2}+\sigma^{2} \frac{d \ln \rho}{d \ln r}
$$

If $\sigma$ is also independent of height $z$ above the disk's equatorial plane, the vertical density distribution is given by the vertical hydrostatic Spitzer solution (Spitzer 1942; Binney \& Tremaine 08, page chapter 4, p. 390)

$$
\rho(z)=\rho_{0} \operatorname{sech}^{2}(z / h)
$$

with $\rho_{0}(\mathrm{r})$ the density in the midplane $(\mathrm{z}=0)$ at radius $\mathrm{r}$ and

$$
h=\frac{\sigma}{\sqrt{2 \pi G \rho_{0}}}
$$

the scale height. The total mass surface density $\Sigma(r)$ of such a disk is (Binney \& Tremaine 2008)

$$
\Sigma=2 \rho_{0} h
$$

so that

$$
\rho_{0}=\frac{\pi G \Sigma^{2}}{2 \sigma^{2}}
$$

The equations 5-7 offer an interesting future observational test of our assumptions as for an exponential disk (equation 10) with constant velocity dispersion the scale height h should increase exponentially with radius

$$
h=\frac{\sigma^{2}}{\pi G \Sigma_{0}} \exp \left(\frac{r}{r_{d}}\right)
$$

Inserting $\rho_{0}$ from equation 7 into equation 3 , the rotation curve in the equatorial plane of a pressurized gas disk is

$$
v_{r o t}^{2}=v_{0}^{2}+2 \sigma^{2} \frac{d l n \Sigma}{d l n r}
$$


For example, for an exponential disk profile with scale length $r_{d}$

$$
\Sigma(r)=\Sigma_{0} \times \exp \left(-\frac{r}{r_{d}}\right)
$$

Equation 9 leads to

$$
v_{\text {rot }}^{2}=v_{0}^{2}-2 \sigma^{2}\left(\frac{r}{r_{d}}\right)
$$

Note that $\mathrm{v}_{\text {rot }}$ is the actually observable rotational velocity of the gas, while $\mathrm{v}_{0}$ is the rotation expected if pressure effects are negligible $(\sigma=0)$. For $v_{r o t} / \sigma \lesssim 3$ the rotational velocity is significantly reduced by turbulent pressure effects for $r \gtrsim r_{d}$. A very similar equation holds for stellar disks as a special form of the Jeans equation (Binney \& Tremaine 2008). Equation 11 was derived for an exponential surface density distribution and a constant velocity dispersion. In general, the situation is more complex as parts of the disk might have constant surface densities while other parts might show a steep gradient. In addition, the velocity dispersion might change with radius. In this case one would have to solve equation (1) directly. The best-resolved high-redshift disk galaxies show roughly constant velocity dispersion profiles (Genzel et al. 2008) and exponentially decreasing $\mathrm{H} \alpha$-surface brightness distributions of the star-forming gas with scale lengths similar to the stellar disks within 1-3 disk scale lengths (Cresci et al. 2009; Bouché et al., in preparation). This is also the region where the rotation curves are flat and achieve their maximum values $v_{\max }$ (Fig. 3). $v_{\max }$ will be used in the following to compare the observations with theory.

For the purpose of this analyses we adopt equation 11 in order to calculate the pressure corrected rotation curves. For simplicity we will also assume that gas and stars have similar disk scale lengths, equal to the sizes as derived for the star-forming gas from the $\mathrm{H} \alpha$ measurements. Note however that there are theoretical reasons why the scale radius of the gaseous disk component, including the part that is not forming stars violently, should be larger than the scale length of the stellar disk (e.g. Sales et al. 2009; Dutton et al. 2010, Guo et al. 2010). This could systematically

bias the derived rotational properties of the disk if the mass fraction of the extended gaseous component is large.

\section{Galactic Disk Model}

We adopt the model by Mo, Mao \& White (1998) of an exponential disk, embedded in a NFW (Navarro et al. 1997) dark matter halo with density distribution

$$
\rho_{D M}(r)=\frac{4 \rho_{c}}{\left(r / r_{s}\right)\left(1+r / r_{s}\right)^{2}}
$$


where $r_{s}$ is the halo scale radius and $\rho_{c}$ is the dark matter density at $r_{s}$. The scale radius is related to the virial radius $r_{200}$ via $r_{s}=r_{200} / c$ where $c$ is the halo concentration parameter. The dark halo rotation curve corresponding to equation 12 is

$$
v_{D M}^{2}(r)=V_{200}^{2}\left(\frac{r_{200}}{r}\right) \frac{\ln \left(1+r / r_{s}\right)-\left(r / r_{s}\right) /\left(1+r / r_{s}\right)}{\ln (1+c)-c /(1+c)}
$$

High-resolution numerical Cold dark matter (CDM) simulations (e.g. Zhao et al. 2009) show that c depends strongly on cosmological redshift. While c decreases with halo virial mass $M_{200}$ at low redshifts, the concentration is roughly constant with $c \approx 4$ and independent of halo mass at $z \approx 2$. The halo virial parameters $r_{200}$ and $M_{200}$ are related to each other through the virial velocity $V_{200}$ (Mo, Mao \& White 1998)

$$
r_{200}(z)=\frac{V_{200}(z)}{10 H(z)}, \quad M_{200}(z)=\frac{V_{200}^{3}(z)}{10 G H(z)}
$$

$H$ is the Hubble parameter that depends on cosmological redshift $\mathrm{z}$ :

$$
H=H_{0}\left[\Omega_{\Lambda}+\left(1-\Omega_{\Lambda}-\Omega_{M}\right)(1+z)^{2}+\Omega_{M}(1+z)^{3}\right]^{1 / 2} .
$$

We adopt a standard $\Lambda$ CDM cosmology with $H_{0}=73 \mathrm{~km} / \mathrm{s} / \mathrm{Mpc}, \Omega_{M}=0.238$ and $\Omega_{\Lambda}=0.762$.

The galactic disk is assumed to follow an exponential surface density profile (equation 10). Its surface density at $r=0, \Sigma_{0}$, is determined by the total disk mass $M_{d}=m_{d} \times M_{200}$ with $m_{d}$ the disk mass fraction of the galaxy

$$
\Sigma_{0}=m_{d} \frac{M_{200}}{2 \pi r_{d}^{2}}
$$

The circular velocity curve of an exponential disk is (Freeman 1970)

$$
v_{d i s k}^{2}(r)=4 \pi G \Sigma_{0} r_{d} y^{2}\left[I_{0}(y) K_{0}(y)-I_{1}(y) K_{1}(y)\right]
$$

with $y=r /\left(2 r_{d}\right)$ and the $I_{n}$ and $K_{n}$ denoting the modified Bessel functions (Binney \& Tremaine 2008).

We will neglect a bulge because we are interested in the outer disk parts where the bulge contribution to the rotation curve is in general negligible. In addition, several of the best resolved SINS galaxies show no evidence for the presence of a significant bulge component (Genzel et al. 2008). In this case and including adiabatic contraction (Blumenthal et al. 1986; Jesseit et al. 2002) of the dark halo, the zero-pressure rotation curve is determined from the implicit equation 


$$
\begin{aligned}
v_{0}^{2}(r) & =v_{d i s k}^{2}(r)+v_{D M}^{2}\left(r^{\prime}\right) \\
r^{\prime} & =r\left[1+\frac{r \times v_{d i s k}^{2}(r)}{r^{\prime} \times v_{D M}^{2}\left(r^{\prime}\right)}\right] .
\end{aligned}
$$

Given $v_{0}(r)$ the pressure-corrected rotation curve $v_{r o t}(r)$ can be calculated from equation 2 or 11 . This is easily done in an iterative process. One first determines the disk rotation curve, neglecting pressure as discussed in MMW. Adopting a value of $v_{\max } / \sigma$, its maximum rotational velocity provides a first guess for $\sigma$ which leads to a revised rotation curve and a corresponding new value of $\mathrm{v}_{\max }$ and $\sigma$. We find that this procedure converges quickly after 10-20 iterations. In the following we will call $v_{r o t}(r)$ the pressure corrected MMW rotation curve. It can be compared directly with observations (section 4.2). In addition we can calculate the total disk angular momentum $J_{d}=2 \pi \int \Sigma v_{\text {rot }} r d r$ that will be used in the next section in order to derive the disk spin parameter $\lambda_{d}$.

\section{Angular Momentum and Baryon Content of High-Z Galaxies}

Figure 1 shows the half-light radii $r_{1 / 2}$ of the SINS high-redshift galaxies versus their maximum rotational velocity $v_{\max }$. The data points and potential uncertainties are discussed in FörsterSchreiber et al. (2009), Cresci et al. (2009) and Law et al. (2009). The errors in $r_{1 / 2}$ and $v_{\max }$ are of order $1-2 \mathrm{kpc}$ and $20-30 \mathrm{~km} / \mathrm{s}$, respectively. We take $r_{1 / 2}$ instead of the exponential disk scale length as it is independent of any assumption about the light profile. $v_{\max }$ is in general a good approximation of the disk's rotational velocities outside of $r_{1 / 2}$. The SINS galaxies segregate

strongly into two distinct classes at a critical value of $v_{\max } / \sigma \approx 3$. We therefore empirically define dispersion-dominated galaxies (open triangles and stars in figure 1) as objects with $v_{\max } / \sigma \leq 3$. For these galaxies turbulent pressure gradients have to be included in the interpretation of the rotation curve. In contrast, for rotation-dominated galaxies (filled triangles), defined by $v_{\max } / \sigma>3$, pressure effects are small. Note that $\sigma$ refers to the intrinsic velocity dispersion in the disk, not to the observed line-of-sight or galaxy-integrated dispersion. Figure 1 shows that most of the dispersion-dominated galaxies have radii of order $1-3 \mathrm{kpc}$ while the radii of rotationally dominated galaxies are on average a factor of 2-3 larger. In addition, the dispersion-dominated systems have rotational velocities of order $100 \mathrm{~km} / \mathrm{s}$ while rotation-dominated galaxies rotate with $250 \mathrm{~km} / \mathrm{s}$.

The specific angular momentum of a dark halo is usually specified by the dimensionless spin parameter (Bullock et al. 2001; Burkert 2009)

$$
\lambda=\frac{J_{200}}{\sqrt{2} M_{200} V_{200} r_{200}}
$$

where $J_{200}$ is the total angular momentum of the halo. $\lambda$ follows a log-normal distribution with a median of $\lambda=0.035$ and a dispersion of 0.55 (Bullock et al. 2001; Hetznecker \& Burkert 2006). 
Cosmological simulations indicate that in the early phases of protogalactic collapse the gas and dark matter are well mixed, acquiring similar specific angular momenta (Peebles 1969; Fall \& Efstathiou 1980; White 1984). If angular momentum were conserved during gas infall and all the gas would settle into the disk, the resulting disk's specific angular momentum $J_{d} / M_{d}$ would be similar to the specific angular momentum of the surrounding dark halo. We cannot measure $\lambda$ directly, but instead can estimate the disk spin parameter, defined as

$$
\lambda_{d}=\frac{J_{d}}{\sqrt{2} M_{d} V_{200} r_{200}}=\lambda \frac{j_{d}}{m_{d}}
$$

with $\mathrm{j}_{d} \equiv \mathrm{J}_{d} / \mathrm{J}_{200}$. If the specific angular momentum of the infalling gas and the resulting disk is equal to the dark halo's specific angular momentum it follows that $\lambda_{d}=\lambda$.

Numerical simulations of galaxy formation find substantial angular momentum loss of the infalling gas component (for a review see e.g. Burkert, 2009). Its origin is not completely clear up to now and might be attributed to numerical problems or missing physics (for a review see e.g. Mayer et al. 2008). If the numerical calculations are however correct, galactic disks should have specific angular momenta and values of $\lambda_{d}$ that are smaller than those of dark matter halos, i.e. on average $\lambda_{d} \leq 0.035$.

In the following we will investigate disk properties for redshift $\mathrm{z}=2.2$ galaxies with dark halo concentrations of $\mathrm{c}=4$ and for given values of $\lambda_{d}, m_{d}$ and $v_{\max } / \sigma$. We start with a first guess of the dark matter virial mass (typically $M_{200}=10^{12} \mathrm{M}_{\odot}$ ). Given $m_{d}$ and by this $\mathrm{M}_{d}$ and assuming a disk scale radius $\mathrm{r}_{d}$ and a $v_{\max } / \sigma$ the procedure discussed in the previous section gives the corresponding disk rotation curve and by this the corresponding $\lambda_{d}$. In an additional iterative step $\mathrm{r}_{d}$ is now varied till the required value of $\lambda_{d}$ is achieved.

Red dashed lines in figure 1 show the standard MMW model predictions without correcting for pressure effects for a given disk spin parameter $\lambda_{d}$ (red labels), adopting a high disk mass fraction, equal to the cosmic baryon fraction $\left(\mathrm{m}_{d}=\mathrm{M}_{d} / \mathrm{M}_{200}=0.2\right)$ in the left panel and a low value of $\mathrm{m}_{d}=0.05$ in the right panel. $r_{1 / 2}$ is determined from the known disk scale length: $r_{1 / 2}=1.68 \times r_{d}$. Stars and open triangles correspond to dispersion-dominated systems, filled triangles to rotationdominated galaxies. Here we assume that the observed half-light radius, traced by $\mathrm{H} \alpha$, is similar to the half-mass radius of the disk. For $\mathrm{m}_{d}=0.2$ especially the rotation-dominated galaxies require very large spin parameters, $\lambda_{d} \approx 0.1-0.2$ which are not in agreement with the theoretical expectations of $\lambda_{d} \leq 0.035$. Adopting $\mathrm{m}_{d}=0.05$ improves the situation considerably. The red dashed lines in the right panel of figure 1 show that, now, MMW models with $\lambda_{d} \approx 0.03-0.07$ fit even the fast rotators, consistent with the upper half of the dark halo $\lambda$ distribution.

The observed baryonic disk masses provide an additional constraint for theoretical models. The symbols in figure 2 show the sum of the stellar mass (from spectral energy distribution analysis) and gas mass (from an application of the Kennicutt-Schmidt star formation relation) of our galaxy sample, plotted as function of $v_{\max }$. McGaugh et al. (2000) and McGaugh (2005) find a remarkably 
tight correlation between baryonic mass and the circular velocity $\mathrm{v}_{\text {circ }}$ at a radius where the rotation curve becomes flat (baryonic Tully Fisher relation): $\mathrm{M}_{d} / \mathrm{M}_{\odot}=50 \times\left(v_{\text {circ }} /(\mathrm{km} / \mathrm{s})\right)^{4}$. Interestingly, the high-redshift data shown in figure 2 deviates strongly from this correlation. The red dashed curves show the expected correlation between disk mass and $v_{\max }$ for different values of $\lambda_{d}$ and for large $\left(\mathrm{m}_{d}=0.2\right.$, left panel $)$ and small $\left(\mathrm{m}_{d}=0.05\right.$, right panel $)$ disk mass fractions according to the MMW model, neglecting pressure effects. According to figure 1 , spin parameters of $\lambda_{d} \approx 0.05$ are required for $\mathrm{m}_{d}=0.05$. The right panel of figure 2 however demonstrates that these values are not consistent with the observed disk masses. On the other hand, the left panels of figure 1 and figure 2 show that large disk mass fractions of $\mathrm{m}_{d}=0.2$ and spin parameters of $\lambda_{d} \approx 0.1-0.2$ are consistent with the observations. While disk mass fractions close to the cosmic baryon fraction are reasonable for these young galaxies where galactic winds might not yet have removed measurable amounts of gas, the required large spin parameters are not consistent with the expectation of $\lambda_{d} \leq \lambda$, as discussed earlier.

\subsection{Dispersion-Dominated Galaxies and the Importance of Pressure Effects}

In section 2 we demonstrated that pressure gradients can significantly affect the rotation curves of galaxies when the ratio or rotational velocity to velocity dispersion, characterized e.g. by $v_{\max } / \sigma$, is sufficiently small. In order to compare the theoretical model with observations we will use the maximum velocity instead of an average velocity, e.g. $v_{2.2}$, measured at 2.15 disk scale lengths. In general this could be dangerous as $v_{\max }$ could occur anywhere in the disk at radii that are not observed. However, as demonstrated by figure 3 for the case of BzK-15504, we find that the rotation curves in general show an extended flat plateau with the maximum at 1-2 disk scale lengths which is in the observed radius regime. In this case, $v_{\max }$ is a good approximation of the typical velocity within the flat part of the rotation curve.

The blue dotted lines in figure 1 show the correlation between $r_{1 / 2}$ and $v_{\max }$ for a MMW model with pressure correction (equation 11), assuming $v_{\max } / \sigma=2$ which is consistent with the dispersion-dominated galaxy sample. The rotation curves are calculated by adopting an exponential disk with a given half-light radius $\mathrm{r}_{1 / 2}=1.68 \times \mathrm{r}_{d}$ (equation 10) and then calculating iteratively the corresponding rotation curve as discussed in section 3 . Note that $v_{\max }$ is now the maximum of the pressure corrected rotation curve which is smaller than the value, neglecting pressure effects. With pressure correction most of the pressure-dominated galaxies lie in the regime $0.02 \leq \lambda_{d} \leq 0.05$ for $\mathrm{m}_{d}=0.2$ and $0.01 \leq \lambda_{d} \leq 0.03$ for $\mathrm{m}_{d}=0.05$ which is in good agreement with theoretical expectations.

A significant pressure contribution reduces significantly $\mathrm{v}_{\max }$ for a given disk mass $\mathrm{M}_{d}$. The blue dotted lines in figure 2 demonstrate this effect. Like in figure 1, they correspond to disks with $v_{\max } / \sigma=2$. Now, the observed masses of pressure-supported SINS galaxies, represented by open triangles, are consistent with spin parameters $\lambda \approx 0.03-0.1$, independent of the adopted value of $\mathrm{m}_{d}$. 
The stars in figure 2 show galaxies with $v_{\max } / \sigma \leq 1.5$. These galaxies are characterised by exceptionally low values of $\mathrm{v}_{\max } \leq 100 \mathrm{~km} / \mathrm{s}$ despite their large masses of $\mathrm{M}_{d} \approx 10^{10} \mathrm{M}_{\odot}$ as expected from equation (11) due to the large pressure contribution.

\subsection{Rotation-Dominated Galaxies and Adiabatic Halo Contraction}

The rotation-dominated SINS sample is characterized by average values of $v_{\max } / \sigma \approx 5$. The problem of unusually high spin parameters and baryon fractions therefore cannot be solved by consideration of pressure gradients. A typical representative of this group is BzK-15504 which has been observed with high angular resolution (Genzel et al. 2006). BzK-15504 is an actively starforming $z=2.4$ galaxy with a stellar disk mass of $10.9_{-0.1}^{+2.7} \times 10^{10} \mathrm{M}_{\odot}$, adopting a Chabrier (2003) initial mass function and a gas mass that varies between $2.8 \pm 0.6 \times 10^{10} \mathrm{M}_{\odot}$ and $4.9 \pm 1.3 \times 10^{10}$ $\mathrm{M}_{\odot}$, depending on the extinction correction applied to the $\mathrm{H} \alpha$ line luminosity (Förster-Schreiber et al. 2009). The total disk mass is then $M_{d} \approx 1.4 \times 10^{11} M_{\odot}$. The radial H $\alpha$ gas surface brightness and the rest-frame optical stellar light distributions are both consistent with an exponential profile with scale length of $4.1 \mathrm{kpc}$.

The observed line width indicates irregular gas motions of $\sigma \approx 45 \pm 20 \mathrm{~km} / \mathrm{s}$ that are constant throughout the disk outside of the central $3 \mathrm{kpc}$ where a bar and an active galactic nucleus (AGN) strongly affect the gas kinematics. The maximum rotational velocity is $v_{\max }=258 \pm 25 \mathrm{~km} / \mathrm{s}$, so that $v_{\max } / \sigma=5.7$. The dark matter virial mass is not well constrained. A minimum value can be inferred if one adopts a disk mass fraction, close to the cosmic baryon fraction $f_{b}=0.2$ : $M_{200} \geq M_{d} / f_{b} \approx 8 \times 10^{11} M_{\odot}$.

The dotted red and blue curves in the left panel of figure 3 show the disk and dark matter rotation curves, respectively, adopting a MMW model with the above mentioned disk parameters and $\mathrm{M}_{200}=8 \times 10^{11} \mathrm{M}_{\odot}$. Both components are equally important with peak rotational velocities at $\mathrm{r} \approx 10 \mathrm{kpc}$ of $250 \mathrm{~km} / \mathrm{s}$ and $280 \mathrm{~km} / \mathrm{s}$. The upper black curve shows the resulting combined rotation curve, neglecting pressure effects. It peaks at $370 \mathrm{~km} / \mathrm{s}$ and is clearly inconsistent with the observations (red open circles) that peak at $\sim 260 \mathrm{~km} / \mathrm{s}$. Including turbulent pressure with $\sigma=45 \mathrm{~km} / \mathrm{s}$ does not significantly change the rotation curve (black points). A test calculation, adopting $\sigma=45 \mathrm{~km} / \mathrm{s}$ shows that increasing $\mathrm{M}_{200}$ (decreasing $\mathrm{m}_{d}$ ) makes the problem even worse while decreasing $\mathrm{M}_{200}$ would require that the galxy has a baryon fraction larger that the cosmic value.

Is this disagreement an observational problem? The uncertainty in the measured disk velocity dispersion is large, of order $20 \mathrm{~km} / \mathrm{s}$. However a dispersion of even $65 \mathrm{~km} / \mathrm{s}$ (dashed black curves in figure 3) makes no big difference. The uncertainties in the determination of the rotation curve are of order $25 \mathrm{~km} / \mathrm{s}$, too small compared with the disagreement of almost $100 \mathrm{~km} / \mathrm{s}$. The only possible solution appears to be a strong reduction of either the baryonic or the dark matter mass within the inner $10 \mathrm{kpc}$. A test calculation shows that the baryonic disk mass would have to be reduced 
by a factor of 2 to $\sim 7 \times 10^{10} M_{\odot}$ for a pressure corrected MMW model with velocity dispersion of $65 \mathrm{~km} / \mathrm{s}$ to fit the observations. The estimates of the stellar and gas masses are subject to many uncertainties and systematics. Spectral energy distribution fitting for BzK-15504 using the Maraston (2005) models yields stellar masses of $M_{*}=9.4(+2.6 /-0.2) \times 10^{10} M_{\odot}$ and gas masses of $M_{\text {gas }}=3.1(+0.6 /-0.6) \times 10^{10} M_{\odot}$ using the Schmidt-Kennicutt relation from Bouché et al. (2007) and the same extinction towards HII regions as towards the stars. The gas masses would be a factor of 2 higher if the extinction towards HII regions is a factor of 2 higher than towards stars (Calzetti et al. 2004). The best-fit Bruzual \& Charlot (2003) model gives $M_{*}=10.9(+2.7 /-0.1) \times 10^{10} M_{\odot}$ and $M_{\text {gas }}=2.8(+0.5 /-0.6) \times 10^{10} M_{\odot}$. Unless the stellar initial mass function is bottom-light or top-heavy it therefore seems difficult to decrease substantially the baryonic disk mass of the galaxy.

Another possibility is a smaller dark halo mass in the disk region. One critical assumption that enters the MMW model is that the dark halo reacts to the formation of the galactic disk by contracting adiabatically. The right panel of figure 3 shows the situation for a MMW model, neglecting dark halo contraction. A comparison with the left panel demonstrates the strong effect of adiabatic contraction. Although the dark matter virial parameters in both cases are the same, without adiabatic contraction the contribution of the dark halo (blue dashed line) within the disk region is small, leading to much better agreement of the model with the observations, especially if we take into account a turbulent pressure, corresponding to a velocity dispersion of $\sigma=65 \mathrm{~km} / \mathrm{s}$ (upper dashed black line), that is still within the observed uncertainties and expected if the disk velocity dispersion tensor would be anisotropic (Aumer et al. 2010).

The problem discussed for BzK 15504 exists for all rotation-dominated galaxies that are represented in the figures 1 and 2 by black filled triangles. Due to their large values of $v_{\max } / \sigma \approx 5$ the effect of pressure gradients is small. The galaxies are therefore represented well by the dashed red lines in both figures which indicate that even for large disk spin parameters $\lambda_{d}=0.1$ the maximum rotational velocity exceeds the observations for given $\mathrm{M}_{d}$. The solid black lines in both figures show the strong effect of neglecting adiabatic dark halo contraction. According to figure 1, the observed disk radi require $\lambda_{d} \approx 0.07$ for $\mathrm{m}_{d}=0.2$ and $\lambda_{d}=0.035$ for $\mathrm{m}_{d}=0.05$. Figure 2 shows that these spin values are also consistent with the observed disk masses.

\section{Origin of Gas Turbulence in High-Redshift Disk Galaxies}

The MMW models also provide insight into the origin of the observed gas turbulence. Let us propose that the main driver of clumpiness and turbulence in gas-rich high-redshift disks is gravitational disk instability. Then we expect gas-rich disks to stay close to the gravitational stability line because of the following reason. A disk that is kinematically too cold with small velocity dispersions is highly gravitationally unstable. Gravitational instabilites generate density and velocity irregularities that drive turbulence and heat the system kinematically. As a result, the gas velocity dispersion increases till it approaches the stability limit where kinetic driving by gravitational instabilities saturates. A disk with even higher velocity dispersions would be stable. 
Here the turbulent energy would dissipate efficiently and the velocity dispersion would decrease again until it crosses the critical velocity dispersion limit where gravitational instabilities become efficient again in driving turbulent motions. In summary, galactic disks should settle close to the gravitational stability line that is determined by the Toomre criterion (Toomre 1964; Wang \& Silk 1994)

$$
Q \equiv \frac{\kappa}{\pi G}\left(\frac{\Sigma_{g}}{\sigma_{g}}+\frac{\Sigma_{*}}{\sigma_{*}}\right)^{-1} \leq Q_{c}
$$

$\kappa$ is the epicyclic frequency that is related to the local angular circular velocity $\Omega$ at radius $\mathrm{r}$ through $\kappa^{2}=r d \Omega^{2} / d r+4 \Omega^{2}$ and $Q_{c}$ is the critical value which is of order unity (Goldreich \& Lynden-Bell 1965; Dekel et al. 2009b). $\Sigma_{*}$ and $\sigma_{*}$ are the stellar surface density and velocity dispersion, respectively. As a test, let us focus again on BzK 15504. As most of the stars in BzK 15504 are likely to have formed during the presently observed star burst we can assume that the stellar velocity dispersion is similar to the observed turbulent gas velocity, i.e. $\sigma_{*} \approx \sigma_{g}$. In addition, the observations show that both components have similar exponential disk scale lengths. If the disk is close to the instability line, its turbulent gas velocity dispersion at any point $\mathrm{r}$ is then

$$
\sigma=\frac{\pi G \Sigma}{\kappa}
$$

where $\Sigma(r)$ is the local baryonic (gas+stars) disk surface density. The lower black dot-dashed curve in the right panel of figure 3 shows the predicted gas velocity dispersion for BzK-15504. It is indeed almost independent of radius and within the uncertainty in agreement with the observed, radially constant value of $45 \pm 25 \mathrm{~km} / \mathrm{s}$. We thus conclude that BzK-15504 is a marginally unstable star-forming disk (Genzel et al. 2006), driven by gravitational instabilities.

We calculated the velocity dispersion profile for all SINS galaxies with $v_{\max } / \sigma \geq 2$ using a pressure corrected MMW model with a dark halo concentration $\mathrm{c}=4$ and neglecting adiabatic halo contraction. The disk mass was taken from the observed stellar and gas masses. The dark halo mass and by this $\mathrm{m}_{d}$ was constrained by fitting the observed maximum velocity of the galaxies. In all cases the theoretically derived velocity dispersion $\sigma_{\text {theo }}$, adopting equation 23 , is almost constant within 1 and 2 disk scale radii. Figure 4 compares the average value of $\sigma_{\text {theo }}$ within 1 and $2 \mathrm{r}_{d}$ with the observed velocity dispersion $\sigma_{o b s}$. Dispersion-dominated (open triangles) and rotation-dominated (filled triangles) systems have similar gas velocity dispersions, indicating that the difference in $v_{\max } / \sigma$ is due to a difference in rotational velocities and not a result of differences in the turbulent gas velocity. Despite the large uncertainties, the theoretical and observed velocity dispersions agree well, strengthening the suggestion that gravitational instabilities are the major driver of turbulence in high-redshift star-forming galaxies. 


\section{Summary and Discussion}

We have shown that pressure gradients in turbulent galactic disks can significantly affect their rotation curves. This effect is well-known for thick stellar disks, leading e.g. to an asymmetric drift of kinematically hot stellar populations in the Galaxy (Binney \& Tremaine 2008). A similar effect is found in models of dust growth in protoplanetary disks where dust particles on ballistic orbits rotate faster than the disk gas which rotates sub-Keplerian due to pressure gradients, leading to fatal dust migration into the central star (e.g. Takeuchi \& Artymowicz 2001).

We analysed the SINS sample of dispersion-dominated high-redshift star-forming galaxies. Including pressure effects and adopting an exponential gas disk with scale length similar to the stellar disk, the models can explain the properties of the dispersion-dominated SINS galaxies very well, with disk spin parameters of $\lambda_{d} \approx 0.025-0.05$ and disk mass fractions of $m_{d} \approx 0.05-0.2$. The strong pressure effect on the structure of dispersion-dominated galaxies depends critically on the assumption of a gas pressure gradient in their disks. The best resolved SINS galaxies indicate a radially constant turbulent velocity dispersion and an exponential decline of the surface density of star-forming gas with scale length similar to the stellar disk (Bouché et al., in preparation). It is however not clear whether all high-redshift galaxy have such a structure. More high-resolution observations are required in order to clarify this point.

For rotation-dominated galaxies, defined by $v_{\max } / \sigma \geq 3$, pressure gradients cannot strongly affect the rotational velocities. Analysing as a test case the galaxy BzK 15504 we showed that its rotation curve allows no significant contribution of dark matter within the visible disk region. This can be achieved with a standard NFW halo that did not contract adiabatically in response to the formation of the galactic disk. The MMW models of the rotation-dominated sample, neglecting adiabatic dark halo contraction, lead to reasonable values of $\lambda_{d} \approx 0.035$ if the disk mass fraction is low $\left(\mathrm{m}_{d} \approx 0.05\right)$ while in the case of high mass fraction $\left(\mathrm{m}_{d} \approx 0.2\right)$, the spin parameters would again be extrem $\left(\lambda_{d} \approx 0.1\right)$.

We assumed that high disk spin parameters of $\lambda_{d} \geq 0.1$ are unreasonable. Scenarios have however been constructed that could lead to disks with higher $\lambda_{d}$ values then their dark matter halos. One of the most promising suggestions are selective galactic outflows of especially lowangular momentum gas from galactic centers (e.g. Maller \& Dekel 2002; Dutton \& van den Bosch 2009).

Sales et al. (09) compared MMW model predictions with the SINS high-redshift disk galaxies and found a good agreement in the rotational velocity versus disk scale length plane (similar to Fig. 1 ) for reasonable spin parameters $\lambda_{d} \approx 0.04-0.06$. They assumed values of $m_{d}=0.05$ and included dark halo contraction. Unfortunately, Sales et al. do not compare their predicted disk masses with observations. In addition, they argue that the observed gas disk radii are a factor $\kappa_{r}=1.8$ larger

than the corresponding stellar disk. They then multiply the disk scale radii resulting from their MMW model for a given $\lambda_{d}$ by this factor in order to compare their model with observations. This implicitly assumes that the gas component has a negligible mass as otherwise it would affect 
$\lambda_{d}$. The assumption of a low gas-to-star fraction is not supported by the SINS observations which typically indicate disk gas fraction of order 30-60\% (Tacconi et al. 2010). In addition, for those cases where stellar data is also available Cresci et al. (2009) find similar scale radii for the stars and gas with $\kappa_{r} \approx 1$, justifying our assumption. Still, more complex multi-component MMW models might be interesting in order to better understand the physical properties of systems with stellar and gaseous disks that are characterised by different scale radii.

The problem that adiabatic halo contraction leads to compact galactic disks with scaling relations that are not in agreement with observations has been discussed for low-redshift galaxies e.g. by Dutton et al. (2007, with references therein) who advocate a model in which the dark halo actually expands rather than contracting. We find that this effect is important also for high-redshift galaxies. Several solutions are currently being discussed. Gnedin et al. (2004) and Gustafsson et al. (2006) argue that the circular orbit adiabatic contraction model (Barnes \& White 1984) considerably overestimates the amount of dark matter contraction. The numerical simulations of Jesseit et al. (2002) however find good agreement with the analytical expression. The dark matter mass fraction in the disk region could also be reduced if one assumes cored dark matter halos (Burkert 1995; Salucci \& Burkert 2000), resulting e.g. from dark matter annihilation, dark matter particle scattering or dynamical interaction. Halo expansion could be triggered by dynamical interaction with massive subclumps or molecular clouds in the disk (El-Zant et al. 2001; Dutton et al. 2007, Mashchenko et al. 2006; Johansson et al. 2009, Abadi et al. 2010; Jardel \& Sellwood 2009) or with bars (e.g. Weinberg \& Katz 2002; Sellwood 2008). Rapid outflows of gas would also lead to halo expansion (Navarro, Eke \& Frenk 1996; Gnedin \& Zhao 2002; Read \& Gilmore 2005).

Whatever the origin, our result demonstrates that the problem of inefficient dark halo contraction is related to the earliest phases of galaxy formation. Although not required in order to produce reasonable spin parameters and disk mass fractions, the processes that suppressed adiabatic halo contraction in rotation-dominated galaxies might also have been active in dispersion-dominated systems. In this case, most dispersion-dominated systems would be characterized by even smaller spin parameters of $\lambda_{d}=0.01-0.03$.

We have analysed the origin of turbulence in high-redshift disk galaxies. Assuming that the disks are marginally unstable we can explain the observed velocity dispersion. This indicates that turbulence is driven and regulated by gravitational instabilites, combined with turbulent energy dissipation. We argued that in this case galactic disks will tend to stay close to a state of marginal gravitational stability which for gas-rich disks corresponds to a velocity dispersion of order 40 $80 \mathrm{~km} / \mathrm{s}$. The energetic source of the turbulent driver is then the potential energy of the disks' gas (Krumholz \& Burkert 2010) which, coupled with viscous forces releases potential energy by spiraling inwards, generating at the end bulge-dominated galaxies as suggested e.g. by Elmegreen et al. (2008) and Dekel et al. (2009a,b). Other energy sources like stellar feedback or accretion energy from infalling gas would then play a minor role because if these processes were dominant the velocity dispersion would likely differ from the value expected for a marginally unstable disk. Note, that within the framework of this scenario the observed velocity dispersion is a signature 
of global gas motions that affect the global disk structure and not just the result of local stellar energy feedback, generating HII regions and driving local outflows of ionized gas. This conclusion is consistent with the finding of Elmegreen \& Elmegreen (2006) that the stellar z-scale heights of highredshift star-forming galaxies are of order $1 \mathrm{kpc}$, which translates to a global velocity dispersion of order $50 \mathrm{~km} / \mathrm{s}$. Whether gas-rich galactic disks naturally evolve towards a state of marginal

stability through gravitational driving of turbulence, combined with turbulent energy dissipation is an interesting question that should be explored in greater details.

Acknowledgments: A.B. is partly supported by a Max-Planck-Fellowship. This work was supported by the DFG Cluster of Excellence "Origin and Structure of the Universe". The Dark Cosmology Centre is funded by the Danish National Research Foundation. We thank A. Dekel, J. Navarro and L. Sales for useful discussions. We thank the referee for a detailed and constructive report that greatly improved the quality of our paper.

\section{REFERENCES}

Abadi, M.G., Navarro, J., Fardal, M., Babul, A. \& Steinmetz, M. 2010, MNRAS, 407, 435

Aumer, M., Burkert, A., Johansson, P. \& Genzel, R. 2010, ApJ, 719, 1230

Barnes, J. \& White, S.D.M. 1984, MNRAS, 211, 753

Binney, J. \& Tremaine, S. 2008, Galactic Dynamics (Princeton, NJ, Princeton University Press, 2008)

Blumenthal, G.R., Faber, S.M., Flores, R. \& Primack, J.R. 1986, ApJ, 301, 27

Bouché, N. et al. 2007, ApJ, 671, 303

Bournaud, F., Elmegreen, B.G. \& Elmegreen, D.M. 2007, ApJ, 670, 237

Bournaud, F. et al. 2008, A\&A, 486, 741

Bruzual, G. \& Charlot, S. 2003, MNRAS, 344, 1000

Bullock, J.S. et al. 2001, ApJ, 555, 240

Burkert, A. 1995, ApJ, 447, L25

Burkert, A. 2009, in IAU Symp. 254, The Galaxy Disk in Cosmological Context, ed. J. Andersen et al. (Cambridge Univ. Press), 437

Calzetti, D., Harris, J., Gallagher, J.S., Smith, D.A., Conselice, C.J., Homeier, N. \& Kewley, L. 2004, AJ, 127, 1403

Chabrier, G. 2003, PASP, 115, 763 
Courteau et al. 2007, ApJ, 671, 203

Cresci, G. et al. 2009, ApJ, 697, 115

Daddi, E. et al. 2004a, ApJ, 600, L127

Daddi, E., Cimatti, A., Renzini, A., Fontana, A., Mignoli, M., Pozetti, L., Tozzi, P. \& Zamorani, G. 2004b, ApJ, 617, 746

Daddi, E. et al. 2007, ApJ, 670, 156

Dekel, A. \& Birnboim, Y. 2006, MNRAS, 368, 2

Dekel, A. et al. 2009a, Nature, 457, 451

Dekel, A., Sari,, R. \& Ceverino, D. 2009b, ApJ, 703, 785

Dib, S., Bell, E. \& Burkert, A. 2006, ApJ, 638, 797

Dutton, A.A., van den Bosch, F.C., Dekel, A. \& Courteau, S. 2007, ApJ, 654, 27

Dutton, A.A. \& van den Bosch, F.C. 2009, MNRAS, 396, 141

Dutton, A.A. et al. 2010, MNRAS in press, astro-ph/1006.3558

Elmegreen, D.M. \& Elmegreen, B.G. 2006, ApJ, 650, 644

Elmegreen, D.M., Elmegreen, B.G., Ravindranath, S. \& Cox, D.A. 2007, ApJ, 658, 763

Elmegreen, B.G,, Bournaud, F. \& Elmegreen, D. 2008, ApJ, 688, 67

Elmegreen, D.M. \& Burkert, A. 2010, ApJ, 712, 294

Elmegreen, B.G., Bournaud, F. \& Elmegreen, D.M. 2008, ApJ, 688, 67

El-Zant, A., Shlosman, I. \& Hoffman, Y. 2001, ApJ, 560, 636

Epinat et al. 2009, A\&A, 504, 789

Erb, D. K., Steidel, C. C., Shapley, A. E., Pettini, M., Reddy, N. A. \& Adelberger, K. L. 2006a ApJ, 646,107

Erb, D. K., Steidel, C. C., Shapley, A. E., Pettini, M., Reddy, N. A. \& Adelberger, K. L. 2006b, ApJ, 647,128

Fall, S.M. \& Efsthatiou, G. 1980, MNRAS, 193, 189

Förster Schreiber, N. M. et al. 2006, ApJ, 645, 1062

Förster Schreiber, N. M. et al. 2009, ApJ, 706, 1364 
Franx, M., et al. 2003, ApJ, 587, L79

Freeman, K.C. 1970, ApJ, 160, 811

Genel, S. et al. 2008, ApJ, 688, 789

Genzel, R. et al. 2006, Nature, 442, 786

Genzel, R. et al. 2008, ApJ, 687, 59

Gnedin, O.Y. \& Zhao, H. 2002, MNRAS, 333, 299

Gnedin, O,Y., Kravtsov, A.V., Klypin, A.A. \& Nagai, D. 2004, ApJ, 616, 16

Goldreich, P. \& Lynden-Bell, D. 1965, MNRAS, 130, 125

Grazian, A. et al. 2007, A\&A, 465, 393

Guo, Q. et al. 2010, submitted to MNRAS, astro-ph/1006.0106

Gustafsson, M., Fairbairn, M. \& Sommer-Larsen, J. 2006, Phys. Rev. D., 74, 3522

Hetznecker, H. \& Burkert, A. 2006, MNRAS, 370, 1905

Jardel, J.R \& Sellwood, J.A. 2009, ApJ, 691, 1300

Immeli, A., Samland, M. Gerhard, O. \& Westera, P. 2004a, A\&A, 413, 547

Immeli, A., Samland, M. Westera, P. \& Gerhard, O. 2004b, ApJ, 611, 20

Jesseit, R., Naab, T. \& Burkert, A. 2002, ApJ, 571, 89

Johansson, P.H., Naab, T.\& Ostriker, J.P. 2009, ApJ, 697, L38

Khochfar, S. \& Silk, J. 2009, ApJ, 700, L21

Kereš D., Katz, N., Weinberg, D.H. \& Davé, R. 2005, MNRAS, 363, 2

Krumholz, M. \& Burkert, A. 2010, ApJ, 724, 895

Law, D., Steidel, C.C., Erb, D.K., Larkin, J.E., Pettini, M., Shapley, A.E. \& Wright, S.A. 2007, ApJ, 669, 929

Law, D. et al. 2009, ApJ, 697, 2057

Maller,A.H. \& Dekel, A. 2002, MNRAS, 335, 487

Maraston, C. 2005, MNRAS, 362, 799

McGaugh, S.S., Schombert, J.M., Bothun, G.D. \& de Blok, W.J.G. 2000, ApJ, 533, L99 
McGaugh, S.S. 2005, ApJ, 632, 859

Mo, H.J., Mao, S. \& White, S.D.M. 1998, MNRAS, 295, 319

Mo,H.J. \& Mao, S. 2000, MNRAS 318, 163

Mashchenko, S., Couchman, H.M.P. \& Wadsley, J. 2006, Nature, 442, 7102

Navarro, J.F., Eke, V.R. \& Frenk, C.S. 1996, MNRAS 283, L72

Mayer, L., Governato, F. \& Kaufmann, T. 2008, ASL, 1, 7

Navarro, J.F., Frenk, C.S. \& White, S.D.M. 1997, ApJ, 490, 493

Noeske, K.G. et al. 2007, ApJ, 660, L43

Ocvirk, P., Pichon, C. \& Teyssier, R. 2008, MNRAS, 390, 1326

Peebles, P.J.E. 1969, ApJ, 155, 393

Pérez-Gonzalez, P.G. et al. 2008, ApJ, 675, 234

Read, J.I. \& Gilmore, G. 2005, MNRAS, 356, 107

Reddy, N. A., Erb, D. K.,Steidel, C. C., Shapley, A. E., Adelberger, K. L., \& Pettini, M. 2005, ApJ, 633, 748

Rudnick, G. et al. 2006, ApJ, 650, 624

Sales, L.V. et al. 2009, MNRAS, 399, L64

Salucci, P. \& Burkert, A. 2000, ApJ, 537, 9

Sellwood, J.A. 2008, ApJ, 679, 379

Shapiro, K.L. et al. 2008, ApJ, 682, 231

Shapley, A.E. et al. 2005, ApJ, 626, 698

Spitzer, L. 1942, ApJ, 95, 329

Stark et al. 2008, Nature, 455, 775

Steidel, C.C., Giavalisco, M., Pettini, M., Dickinson, M. \& Adelberger, K.L. 1996, ApJ, 462, L17

Steidel, C.C., Shapley, A.E., Pettini, M., Adelberger, K.L., Erb, D.K., Reddy, N.A., \& Hunt, M.P. 2004, ApJ, 604, 534

Tacconi, L. et al. 2010, Nature, 463, 781 
Takeuchi, T. \& Artymowicz, P. 2001, ApJ, 557, 990

Toomre, A. 1964, ApJ, 139, 1217

van Dokkum, P.G. et al. 2006, ApJ, 638, L59

Van Starkenburg, L., van der Werf, R.P., Franx, M., Labb’e, I., Rudnick, G. \& Wuyts, S. 2008, A\&A, 488, 99

Wang, B. \& Silk, J. 1994, ApJ, 427, 759

Weinberg, M.D. \& Katz, N. 2002, ApJ, 580, 627

White, S.D.M. 1984, MNRAS, 286, 38

Wright, S.A. et al. 2007, ApJ, 658, 78

Wright, S.A. et al. 2009, ApJ, 699, 421

Zhao, D.H., Jing, Y.P., Mo, H.J. \& Boerner, G. 2009, ApJ, 707, 354 


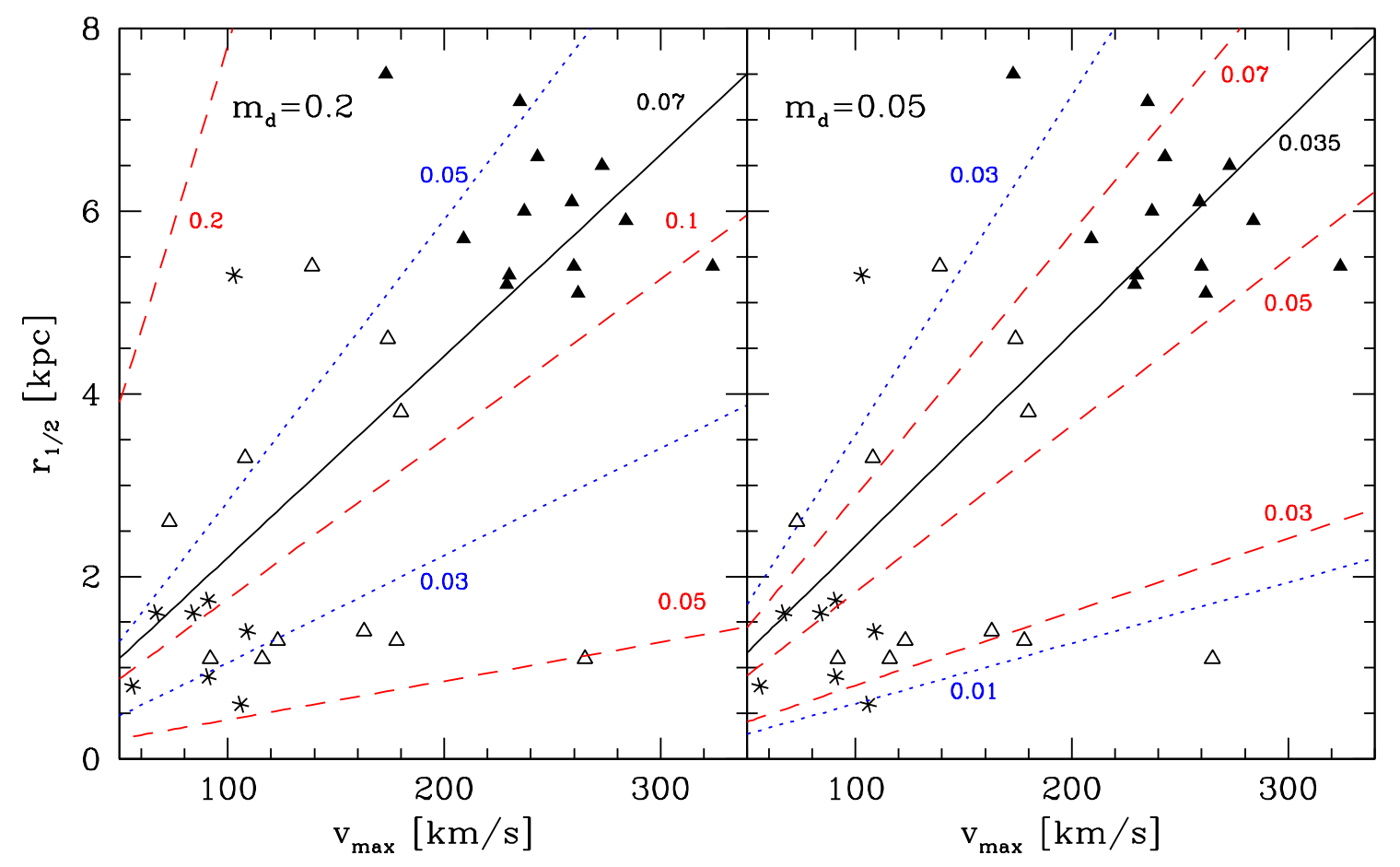

Fig. 1. - The left and right panels show the disk half-light radii $r_{1 / 2}$ versus the maximum rotational velocities $v_{\max }$ of models with disk mass fractions of $\mathrm{m}_{d}=0.2$ and $\mathrm{m}_{d}=0.05$, respectively and compare them with the SINS high-redshift disk sample. Open triangles correspond to dispersion-dominated galaxies, filled triangles to rotation-dominated objects. Stars show extremely dispersion-dominated systems with $\mathrm{v}_{\max } / \sigma \leq 1.5$. Red dashed lines show the theoretically predicted correlation between $r_{1 / 2}$ and $v_{\max }$ if pressure effects are neglected for various values of the disk spin parameter $\lambda_{d}$ (red labels) Blue dotted lines show MMW models including the effect of a pressure gradient and adopting $v_{\max } / \sigma=2$. The black solid lines represent rotation-dominated galaxies with $v_{\max } / \sigma=5$, neglecting adiabatic dark halo contraction. 


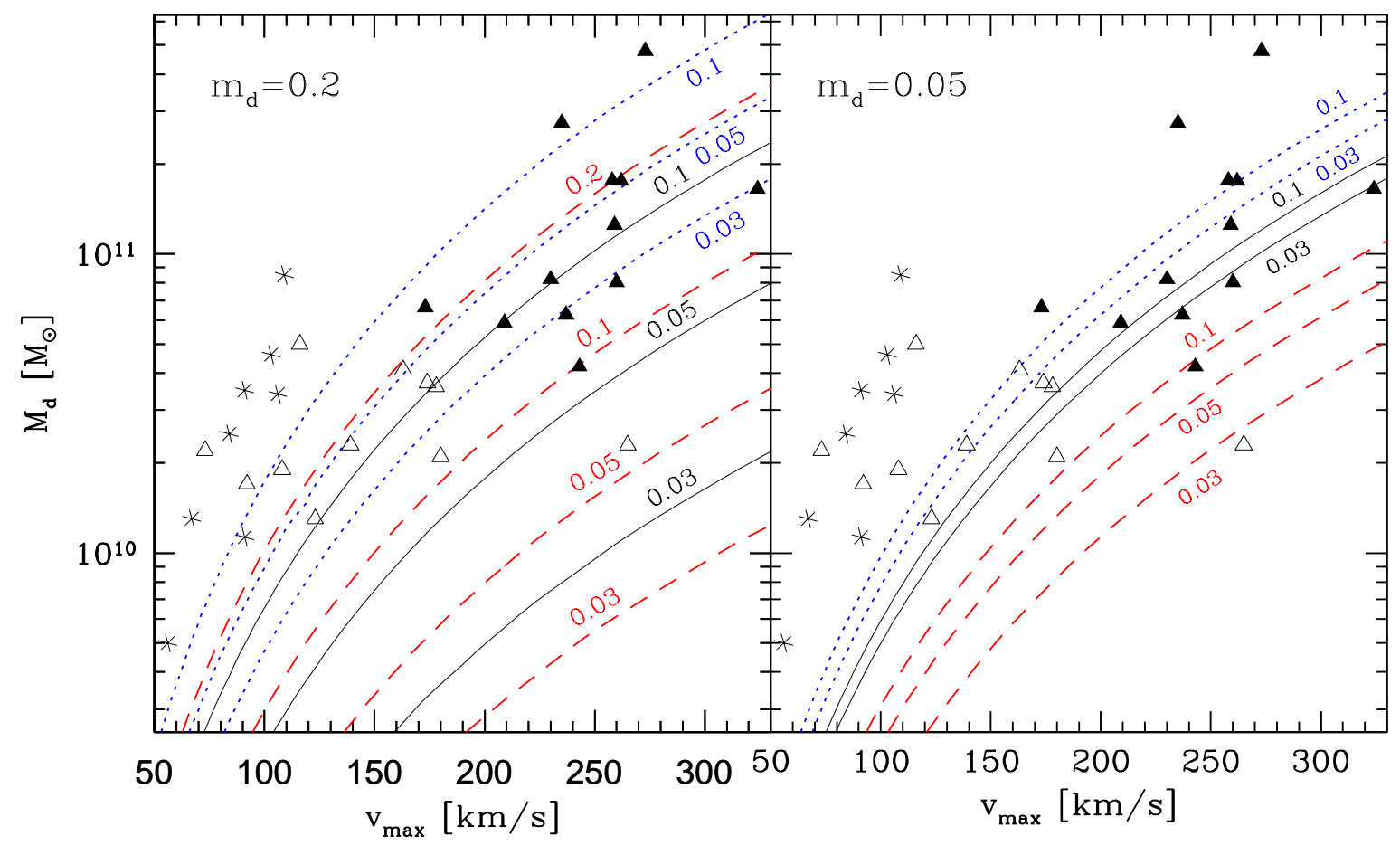

Fig. 2.- Open and filled triangles show the observationally inferred disk masses versus the maximum velocity of dispersion-dominated and rotation-dominated high-redshift SINS galaxies, respectively. The stars show extremely dispersion-dominated galaxies with $v_{\max } / \sigma \leq 1.5$. The red dashed curves show the predictions of the standard MMW model, adopting a disk mass fraction of $\mathrm{m}_{d}=0.2$ in the left panel and $\mathrm{m}_{d}=0.05$ in the right panel. Red labels indicate the corresponding disk $\lambda_{d}$ parameters. The blue dotted lines show the situation if turbulent pressure is taken into account, adopting $v_{\max } / \sigma=2$. Black, solid curves correspond to MMW models with $v_{\max } / \sigma=5$, neglecting adiabatic contraction of the dark halo. 


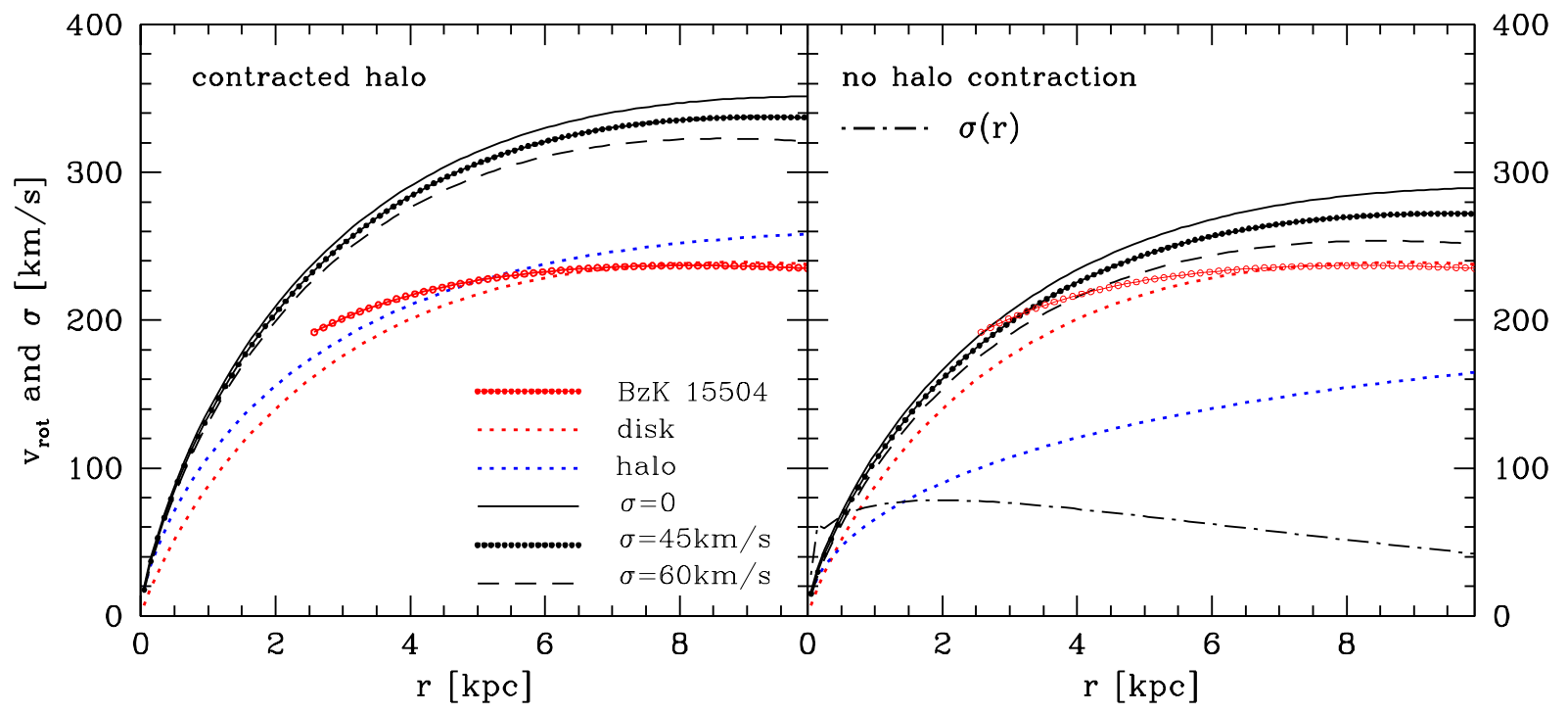

Fig. 3.- Open red circles in both panels show the inclination and resolution corrected intrinsic rotation curve of BzK-15504, inferred from fitting the observed two-dimensional distribution of $\mathrm{H} \alpha$ velocities (Genzel et al. 2006). We focus on the rotational properties outside of $3 \mathrm{kpc}$ as the inner regions are affected by the central AGN and bar. The dotted red and blue lines in the left panel show the theoretically expected contribution of the disk and dark halo component, respectively, adopting a cosmic baryon fraction $\mathrm{m}_{d}=\mathrm{f}_{b}=0.2$ and an adiabatically contracted dark halo with concentraction $\mathrm{c}=4$. The combination of both curves leads to the zero-pressure total rotation curve (upper black line) that exceeds the observed maximum rotational velocity by more than $100 \mathrm{~km} / \mathrm{s}$. The black points and the dashed black curve correspond to the pressure corrected rotation curve, including pressure effects with a gas velocity dispersion of $45 \mathrm{~km} / \mathrm{s}$ and $65 \mathrm{~km} / \mathrm{s}$, respectively. The right panel shows the situation without adiabatic dark halo contraction. Symbols and lines are the same as in the left panel. Now the resulting rotation curve is in much better agreement with the observations. The lower dot-dashed black curve shows the theoretically predicted velocity dispersion profile assuming a constant Toomre stability parameter of $\mathrm{Q}=1$. 


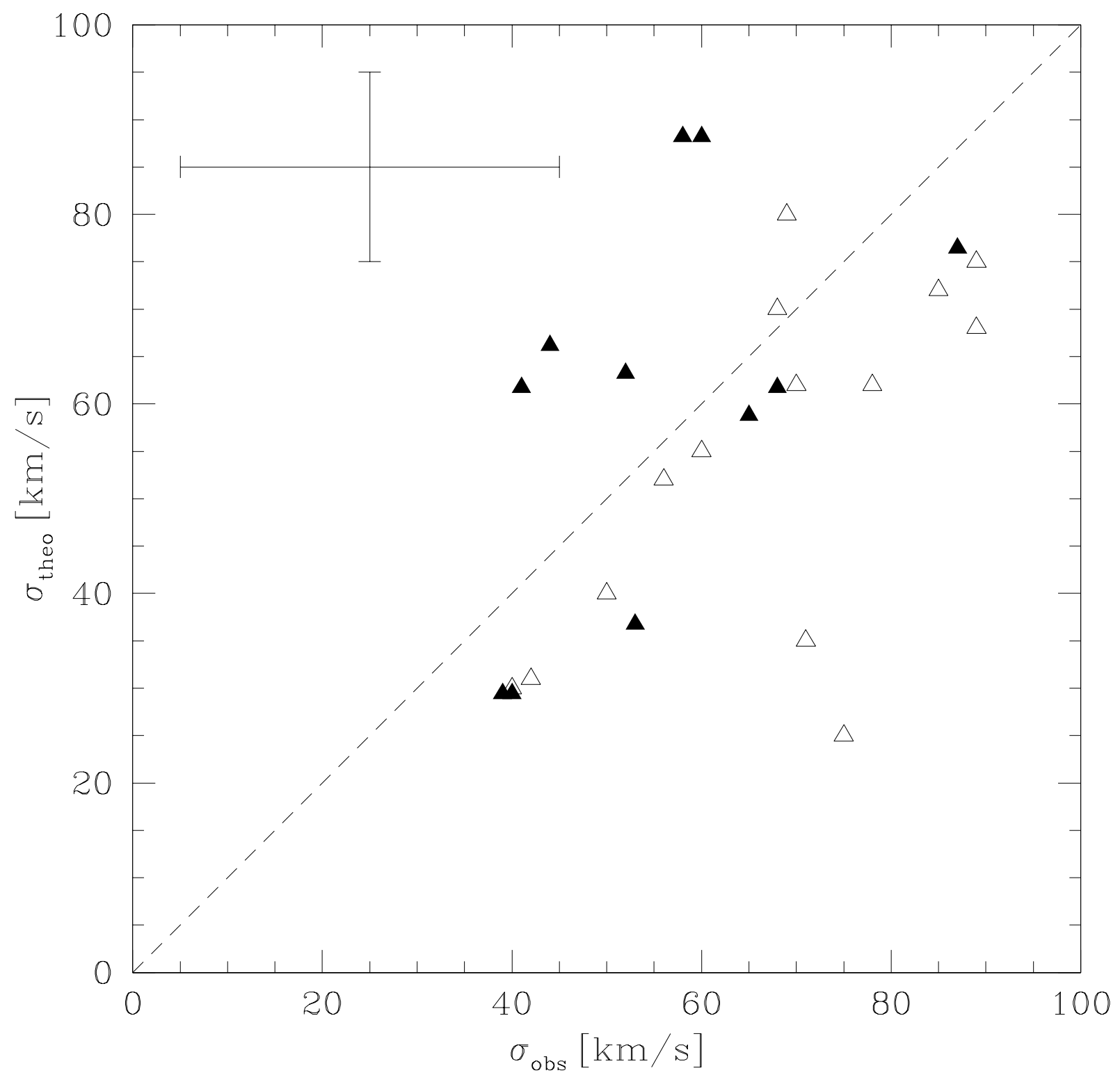

Fig. 4.- The observed velocity dispersion $\sigma_{o b s}$ of the SINS high-redshift galaxy sample is compared with theoretical expectations $\sigma_{\text {theo }}$, adopting a pressure-corrected MMW model without adiabatic halo contraction. $\sigma_{\text {theo }}$ was calculated by averaging the velocity dispersion profile between one and two disk scale lengths. Open and filled triangles correspond to dispersion and rotation-dominated systems, respectively. The error bar in the upper left corner indicates the observational uncertainties. 